\title{
Aerobic Exercise and Eucalyptus Globulus: Cardio- Affective Effects on Recovery and Stress
}

\author{
Alfredo A Teixeira-Araujo ${ }^{1,2}$, Jackson R G S Almeida ${ }^{1,3}$, \\ Loumaíra C Cruz ${ }^{1,4}$, Xirley P Nunes ${ }^{3}$, Sérgio R Moreira ${ }^{1,2, *}$ \\ ${ }^{1}$ Graduate Program Biological and Health Sciences, Federal University of Vale do \\ São Francisco, Petrolina, Brazil \\ ${ }^{2}$ Graduate Program on Physical Education, Federal University of Vale do São \\ Francisco, Petrolina, Brazil \\ ${ }^{3}$ Graduate Program in Natural Resources in the Semiarid, Federal University of \\ Vale do São Francisco, Petrolina, Brazil \\ ${ }^{4}$ College of Physical Education, University Center Leão Sampaio, Lagoa Seca, \\ Brazil \\ * Corresponding author: Sérgio R. Moreira, Federal University of São Francisco Val- \\ ley, UNIVASF, Physical Education Collegiate, Av. Jose de Sá Maniçoba, Petrolina, \\ Brazil.Tel:+55-8738625017,E-mail:sergio.moreira@univasf.edu.br
}

DOI: $10.21859 / \mathrm{ijcp}-020101$

\section{Submited: 10.06 .2016 \\ Accepted: 10.30 .2016 \\ Keywords: \\ Endurance Exercise \\ Eucalyptus Essencial Oil \\ Psychophysiological \\ Responses}

(C) 2016. International Journal of Cardiovascular Practice.

\begin{abstract}
Introduction: It is necessary to investigate the interrelation between physical exercise and aromatherapy, regarding cardiovascular and affective functions of human. Thus, the aim of this study was to evaluate the effects of aerobic exercise (AE) and eucalyptus essential oil (EO) in cardiovascular and affective responses.

Methods: Twelve adults (19-39 years) performed in a randomized order in different days: 1. AE session; 2. Inhalation of EO session; 3. AE + EO session and; 4. Control session. In each session remained at rest over $15 \mathrm{~min}$ plus $5 \mathrm{~min}$ to inhaling EO or blank air in Control session. The $\mathrm{AE}$ was $15 \mathrm{~min}$ run/walk at $80-85 \%$ of maximum heart rate. After recovery (30 min) the Cold Pressor Test (CPT) was applied. At rest, recovery and during CPT were measured cardiovascular and affective variables.

Results: The variation of systolic blood pressure in the CPT was attenuated by AE + EO $(12.3 \pm 14.9 \mathrm{mmHg})$ and $\mathrm{EO}(12.6 \pm 6.7 \mathrm{mmHg})$ compared to Control $(17.3 \pm 6.5$ $\mathrm{mmHg} ; \mathrm{P}<0.05) . \mathrm{AE}+\mathrm{EO}$ increased pleasure in recovery $\left(1^{\prime}=1.2 \pm 1.9 \mathrm{pts}\right.$ and $30^{\prime}=0.6$ $\pm 1.1 \mathrm{pts})$ and reduced displeasure in stress $(-3.4 \pm 1.7 \mathrm{pts})$ when compared to Control in recovery $\left(1^{\prime}=-0.5 \pm 1.4\right.$ pts and $30^{\prime}=-0.7 \pm 1.5$ pts, respectively; $\left.\mathrm{P}<0.05\right)$ and stress $(-4.6$ \pm 1.8 pts; $\mathrm{P}<0.05)$.

Conclusions: We conclude that aerobic exercise and eucalyptus essential oil attenuated cardiovascular responses and modulated the affect on recovery and stress.
\end{abstract}

\section{INTRODUCTION}

Changes in lifestyle can influence the psychophysiological well-being of an individual, which increases the risk of cardiovascular diseases in the medium to the long-term [1]. Changes in the autonomic nervous system can be observed during exposure to environmental stressors. Under such conditions, increases in the sympathetic nerve activity [2] may reflect on blood pressure and heart-rate hyperreactivity [3]. On the other hand, the individual's immersion in natural environments has been discussed as a strategy for promoting health [4] since many aromatic components exhaled by plants present therapeutic properties [5]. Thus, natural essential oils extracted from plants can be used for medicinal purposes. Eucalyptus globulus is an example of a species that shows special participation of the monoterpenic oxide 1.8-cineole [6]. Eucalyptol (1.8-cineole) has been presented as a cardiovascular system modulator acting on the cardiac inotropism of rats $[7,8]$. In addition to this, some regions of the central nervous system are activated when there is the inhalation of aromatic components derived from essential oils. It shows that a possible action mechanism might start in the olfactory bulb and go up to the hypothalamus, thus influencing the autonomous nervous system [2].

Currently, a $40 \%$ reduction in cardiovascular risks has been related to physical activity practicing. Such practices can modulate vascular and structure functions [9]. According to such perspective, aerobic exercise may play an important role in autonomic control and adjust the hemodynamic functions of individuals recovering from a single session [10]. The aerobic post-exercise hypotension may be associated with the attenuation of the sensitivity to sympathetic stimulation [11, 
12]. Such change has been discussed as an important protective mechanism of the blood pressure reactivity to induced stress in humans [3]. However, little is known about the independent effects of low doses of aerobic exercise in these responses.

Furthermore, the effects of aromatherapy can have an impact on the cognitive function of an individual, therefore influencing the mood and some associated co-variables [13]. Therefore, it is still necessary to investigate the interrelation between physical exercise and aromatherapy, regarding cardiovascular and affective functions of human. Thus, the current study aimed to investigate acute effects of low doses of aerobic exercises associated with eucalyptus essential oil on cardiovascular and affective responses to recovery from the session and during induced stress.

\section{METHODS}

\section{Subjects}

Twelve subjects from both genders ( 8 male, 4 female) participated in the study and it took under consideration a power of $90 \%, \alpha=5 \%$ and four experimental sessions for comparison. All volunteers signed a consent form and the study was approved by the Ethics and Research Committee of Federal University of Vale do São Francisco (Protocol n. 0003/200813).

\section{General Procedures}

The volunteers performed four experimental sessions in different days separated by one week period each. It was done following a randomized order: 1 . Aerobic exercise (AE); 2. Eucalyptus essential oil (EO) inhalation; 3. Combination of EA + EO and; 4. Control. Measurements of blood pressure $(\mathrm{BP})$, heart rate (HR), rate pressure product (RPP) and pleasure or displeasure perception were performed during pre-session resting moments as well as in the recovery periods from the experimental sessions. A blood pressure reactivity test was also applied at the end of individuals' recovery period from the experimental sessions. Fig 1 shows the experimental design.

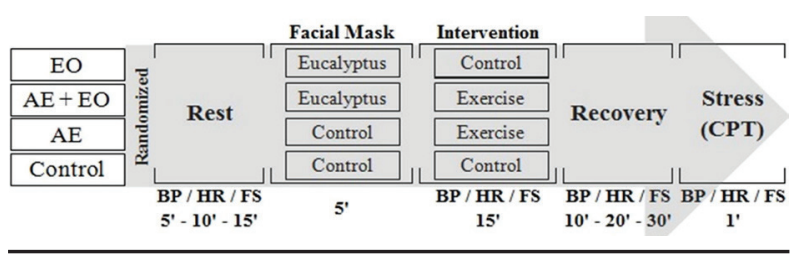

Figure 1: Experimental design. EO, eucalyptus essential oil; AE + EO, aerobic exercise plus Eucalyptus essential oil; AE, aerobic exercise; CONTROL, control session or control period within session; $\mathrm{BP} / \mathrm{HR} / \mathrm{FS} / \mathrm{RPE}$, blood pressure, heart rate, feeling scale and rate of perception effort measurements; CPT, cold pressor test.

\section{Aerobic Exercise (AE)}

The AE session was performed in a cement track. Participants had to run/walk, for 15 minutes, under intensity between 80 $85 \%$ of the maximum HR fixed according to the age of the participants (220-age). At the end of the $\mathrm{AE}$ session, the rate of perceived exertion (RPE) was assessed by means of the 15-point Borg scale [14], in order to obtain additional information on the intensity of the performed exercise. The current study aims to apply moderate exercise intensity - below the anaerobic threshold which corresponds to RPE below $13 / 14$ in the Borg scale [15].

\section{Eucalyptus Essential Oil Inhalation (EO)}

Volunteers used a disposable mask and inhaled three drops of eucalyptus essential oil for 5 minutes, in laboratory environment under controlled temperature of $24-25^{\circ} \mathrm{C}$. Substance inhalation happened immediately prior to $\mathrm{AE}$, in order to complete the $\mathrm{AE}+\mathrm{EO}$ session and also, before the exercise control condition, aiming to complete the EO session alone. A control session was performed but with no $\mathrm{EO}$ or $\mathrm{AE}$ inhalation. It was done in order to complete the four experimental sessions. However, in the moment EO inhalation was performed, volunteers remained in a condition similar to that from other sessions, when they used mask on their faces, thus inhaling blank air.

\section{Extracting Eucalyptus Essential Oil}

Eucalyptus essential oil extraction was performed by hydrodistillation and using the Clevenger system. Five hundred grams $(500 \mathrm{~g})$ of Eucalyptus globulus leaves were fragmented and placed in a volumetric flask fulfilled with water and adapted to the extractor. Such procedure was done in a Pharmacology Laboratory. Leaves were taken to a heating mantle at boiling temperature so that the water vapors and volatiles were driven to the condenser in which the distillation happened [16]. The eucalyptus essential oil was collected in a $2 \mathrm{~mL}$ vial and a small amount of anhydrous sodium sulfate was added to it in order to absorb the water molecules within the oil. Percentages of substances found in the eucalyptus essential oil have been identified through gas chromatography-mass spectrometry (GC-MS) and eucalyptol (1.8-cineole) represented $69.8 \%$ of the sample.

\section{Measurement of Cardiovascular Variables}

Volunteers were taken to an Exercise Physiology Laboratory and seated in a comfortable chair with their left upper limb relaxed at heart level. Systolic BP (SBP), diastolic BP (DBP), HR and $\mathrm{RPP}$ were checked at rest period, before the experiments for 15 minutes $(3 \times 5 \mathrm{~min})$, during the recovery after the experiments for $30 \mathrm{~min}(3 \times 10 \mathrm{~min})$ and during the Cold Pressor Test (CPT), by means of an automated sphygmomanometer (Microlife, BP 3AC1-1, USA) validated by Stergiou et al. [17].

\section{Cardiovascular Stress Test}

After the 30 minutes recovery to each experimental session, participants were submitted to a thermal stress test, following the CPT protocol [18], which was used because of its ability to induce cardiovascular stress [19].

During the CPT, participants voluntarily immersed their right hand in water under controlled temperature between $3-4^{\circ} \mathrm{C}$. During CPT, the individual remained with the hand immersed for one minute, a period used to perform SBP, DBP, HR and RPP reactivity measurements. 


\section{Affective Responses}

The subjects were asked about their perception on pleasure/displeasure from feeling scale [20] in the end of the initial resting period after the experimental sessions, every 10 minutes during the 30 minutes recovery period as well as immediately at the end of the CPT. The Feeling Scale presents an 11-point bipolar measure, ranging from -5 to + 5 and verbal descriptors ranging from "very poor" to "very good" and a "neutral" zero point. Prior to the experimental session, the scale was used to anchor the participants' memory $[21]$.

\section{Statistical Analysis}

Shapiro Wilk Test was previously adopted to test data normality. It was used the descriptive statistics to mean, standard deviation, standard error of the mean and percentage frequency. Intra- and intersession comparisons performed repeated measures by ANOVA test and an LSD Post Hoc test was adopted to detect pairs of differences. Significance level was set at $\mathrm{P}<0.05$. All analyses were performed through the Statistica ${ }^{\circledR}$ software, version .5.1

\section{RESULTS}

The investigated sample $(\mathrm{n}=12)$ had mean age of $26.9 \pm$ 7.5 years old, body mass of $69.1 \pm 16.7 \mathrm{~kg}$, height of $165.9 \pm$ $6.8 \mathrm{~cm}$ and body mass index of $24.9 \pm 4.4 \mathrm{~kg} / \mathrm{m}^{2}$. According to risk stratification, volunteers were considered apparently healthy.

Table 1 presents the results of cardiovascular and affective variables obtained at rest, which did not differ among experimental sessions $(\mathrm{P}>0.05)$.

During interventions, it was possible to see that there were no differences for the $\%$ of maximum HR between sessions with exercise (AE: $84 \pm 1$ vs. $\mathrm{AE}+\mathrm{EO}: 83 \pm 2$; $\mathrm{P}=0.06$ ) and without exercise (EO: $38 \pm 5$ vs. Control: $38 \pm 7$; P $=0.89$ ). There was also no difference in RPE between sessions with exercise (AE: $12.3 \pm 2.4$ vs. $\mathrm{AE}+\mathrm{EO}: 12.1 \pm 2$; $\mathrm{P}=0.72$ ), which demonstrates that the intensity of aerobic exercise was moderate in both sessions.

Figure 2 shows the SBP variation during the experimental sessions. It was found lower reactivity of SBP, during stress, for the $\mathrm{AE}+\mathrm{EO}$ session and the $\mathrm{EO}$ session alone, when compared with the control session $(\mathrm{P}<0.05)$. DBP showed no difference between sessions $(\mathrm{P}>0.05)$.

Figures 4 and 5 show HR and RPP variations. There were no significant differences in any of the periods. However, by descriptively analyzing the $\mathrm{AE}$ session, in relation to the $\mathrm{AE}$ + EO session, it was found that there was a variation of $18 \%$ in HR and RPP reactivity during stress, as well as $40 \%$ less variation in $\mathrm{RPP}$ reactivity in the $\mathrm{EO}$ session in comparison with the control session.

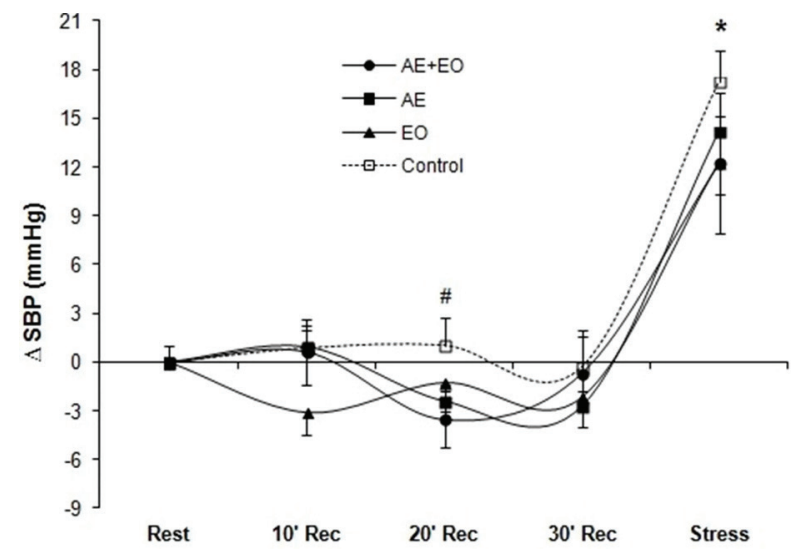

Figure 2: Mean variation ( \pm SEM) of systolic blood pressure (SBP) in different moments (Rest - 10-30min of recovery and Stress) of Control, Eucalyptus essential oil (EO), Aerobic exercise (AE) and $\mathrm{AE}+\mathrm{EO}(\mathrm{n}=12) .{ }^{*} \mathrm{p}<0.05$ to $\mathrm{AE}+\mathrm{EO}$ and $\mathrm{EO}$ session; $\# \mathrm{p}=0.06$ to $\mathrm{AE}+\mathrm{EO}$ session.

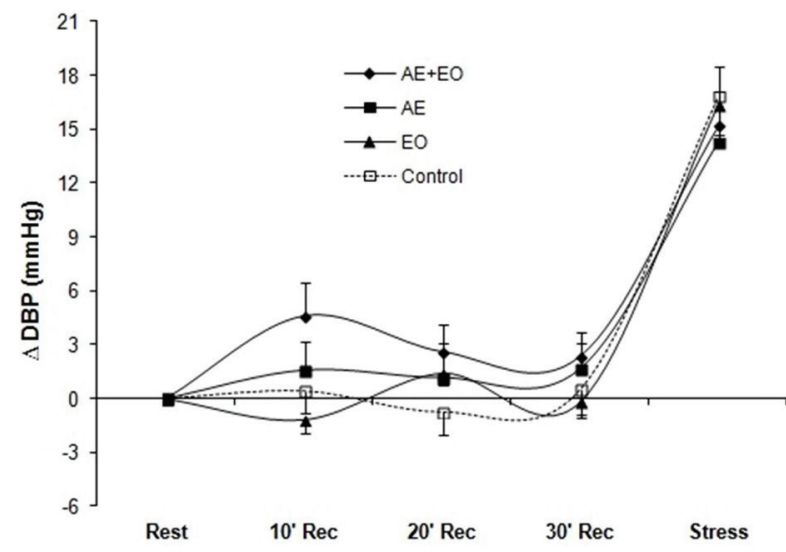

Figure 3: Mean variation ( \pm SEM) of diastolic blood pressure (DBP) in different moments (Rest - 10-30min of recovery and Stress) of Control, Eucalyptus essential oil (EO), Aerobic exercise $(\mathrm{AE})$ and $\mathrm{AE}+\mathrm{EO}(\mathrm{n}=12)$.

\begin{tabular}{|c|c|c|c|c|c|}
\hline & $\mathrm{EO}$ & $\mathrm{AE}+\mathrm{EO}$ & $\mathrm{AE}$ & Control & $\mathrm{P}$ \\
\hline SBP, mmHg & $115 \pm 13$ & $117 \pm 14$ & $118 \pm 11$ & $114 \pm 13$ & $>0.05$ \\
\hline DBP, $\mathbf{m m H g}$ & $73 \pm 9$ & $75 \pm 8$ & $77 \pm 8$ & $74 \pm 9$ & $>0.05$ \\
\hline HR, bpm & $73 \pm 8$ & $77 \pm 11$ & $79 \pm 13$ & $73 \pm 13$ & $>0.05$ \\
\hline RPP, $\mathbf{m m H g} \times \mathbf{b p m}$ & $8382 \pm 1188$ & $8987 \pm 1803$ & $9349 \pm 1726$ & $8308 \pm 1874$ & $>0.05$ \\
\hline FS, Points & $0.3 \pm 0.9$ & $0.2 \pm 1.2$ & $0.7 \pm 1.5$ & $0.5 \pm 1.3$ & $>0.05$ \\
\hline
\end{tabular}

${ }^{a}$ Abbreviations: SBP, systolic blood pressure; DBP, diastolic blood pressure; HR, Heart rate; RPP, rate pressure product; FS, feeling scale. 


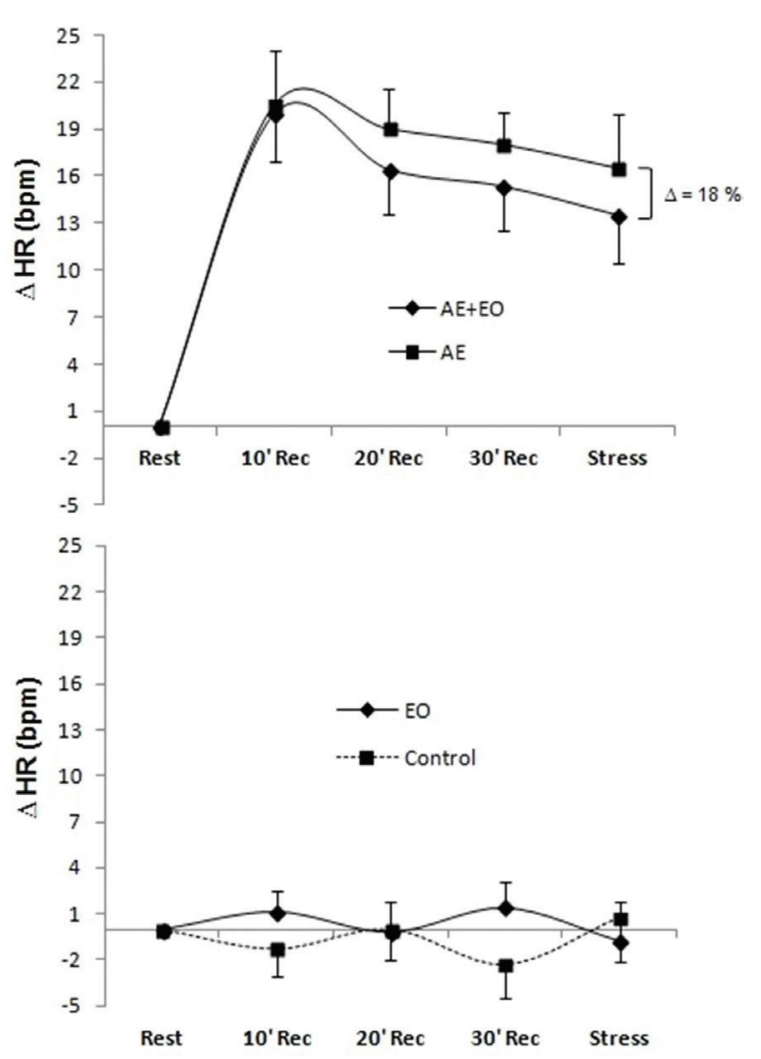

Figure 4: Mean variation ( \pm SEM) of heart rate $(\mathrm{HR})$ in different moments (Rest - 10-30min of recovery and Stress) of Control and Eucalyptus essential oil (EO) on bottom panel and Aerobic exercise $(\mathrm{AE})$ and $\mathrm{AE}+\mathrm{EO}$ on top panel $(\mathrm{n}=12)$.

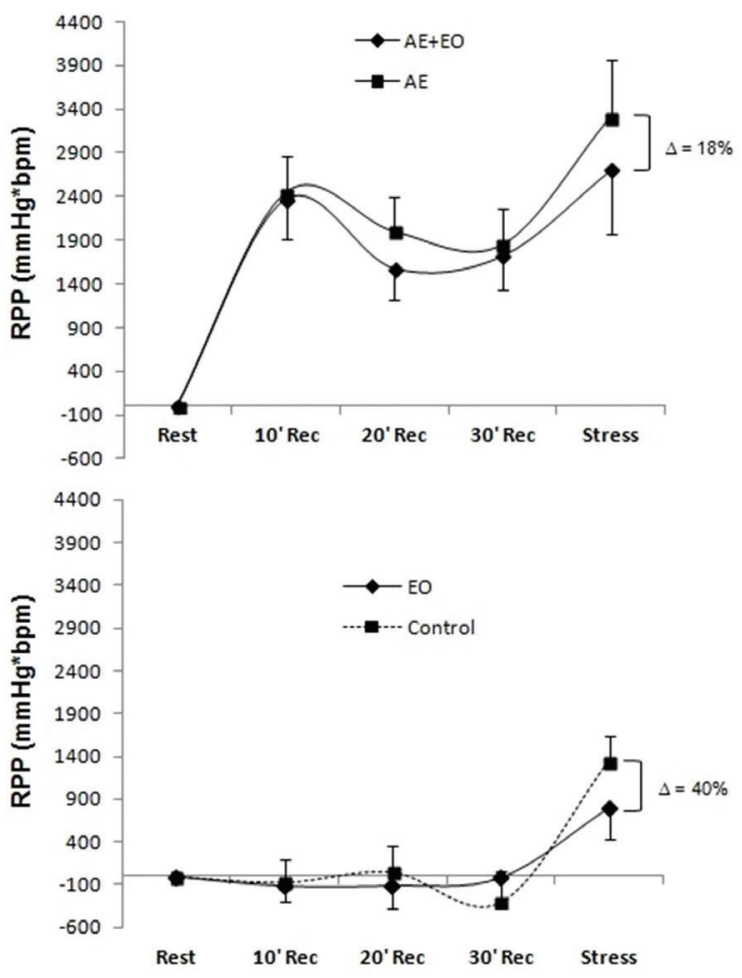

Figure 5: Mean variation ( \pm SEM) of rate pressure product (RPP) in different moments (Rest - 10-30min of recovery and Stress) of Control and Eucalyptus essential oil (EO) on bottom panel and Aerobic exercise $(\mathrm{AE})$ and $\mathrm{AE}+\mathrm{EO}$ on top panel $(\mathrm{n}=12)$.
Figure 6 shows the results for pleasure/displeasure perception among the different experimental sessions. Differences were found between $\mathrm{AE}+\mathrm{EO}$ and the control sessions at the 1 st and 30th minute of recovery as well as during stress $(\mathrm{P}<$ $0.05)$. Differences also occurred at the 1 st minute of recovery, between $\mathrm{EO}$ and the control sessions $(\mathrm{P}<0.05)$, as well as during stress between $\mathrm{AE}+\mathrm{EO}$ and $\mathrm{AE}$ sessions $(\mathrm{P}<0.05)$.

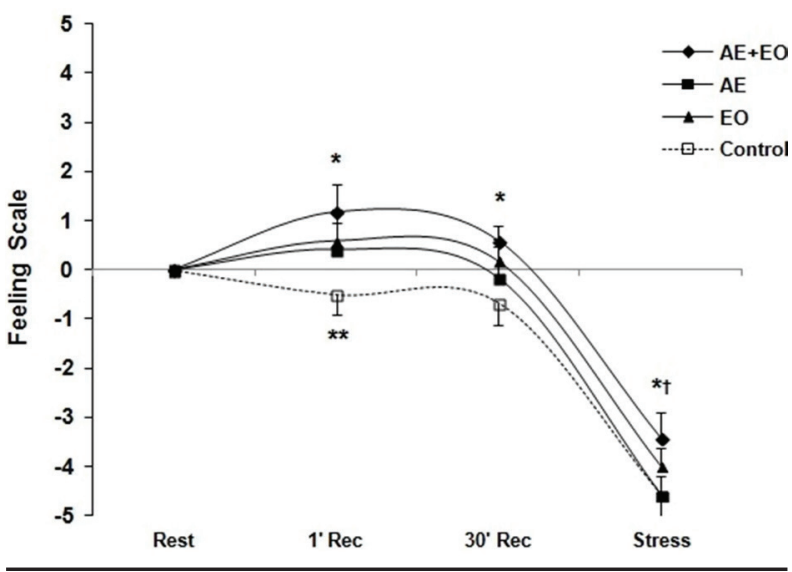

Figure 6: Mean variation ( \pm SEM) of feeling scale in different moments (Rest - 10-30min of recovery and Stress) of Control, Eucalyptus essential oil (EO), Aerobic exercise $(\mathrm{AE})$ and $\mathrm{AE}+\mathrm{EO}(\mathrm{n}=12)$. ${ }^{*} \mathrm{p}<0.05$ to Control session; ${ }^{* *} \mathrm{p}<0.05$ to $\mathrm{EO} ; \mathrm{p}<<0.05$ to $\mathrm{AE}$.

\section{DISCUSSION}

The main findings of the study were that only 15 minutes of aerobic exercise at moderate intensity associated with 5 minutes of eucalyptus essential oil inhalation or the eucalyptus essential oil alone significantly attenuated the SBP reactivity during induced cardiovascular stress (Fig 2). Furthermore, greater sense of pleasure during recovery and less sense of displeasure during stress were modulated after performing a session combining aerobic exercise and eucalyptus essential oil (Fig 6). Although there were no significant results, reductions of $18 \%$ in HR and RPP were observed during stress, after the intervention with aerobic exercise and eucalyptus essential oil inhalation if compared with aerobic exercise (Figs 4 and 5). During cardiovascular stress, RPP was $40 \%$ lower after the intervention with eucalyptus essential oil when compared with the control session (Fig 5).

These findings suggest the important role played by the eucalyptol (1.8-cineole) in acute cardiovascular protection, in the face of stress condition in this system. Soares et al. [8] showed that eucalyptol reduced cardiac inotropism in papillary muscles of rats, fact that can be directly related to SBP, HR and RPP in the current study. Dayawansa et al. [22] demonstrated, via cedrol oil inhalation by humans, a reduced sympathetic nerve activity and an increased parasympathetic tone, thus presenting a consequent increase in the baroreflex sensitivity. Such results can be associated with $\mathrm{Li}$ et al. [23], who found a significant effect of a 2 hours walk in a forest park on BP reduction, corroborating the current outcomes, demonstrating that the interaction between aerobic exercise and eucalyptus essential oil attenuates SBP reactivity to induced stress. However, according to the current study, a 15 minutes run/walk at moderate intensity was enough. Reduced HR and RPP percentages during stress and after 
eucalyptus essential oil inhalation suggest a central action regarding the interaction between the 1.8-cineole component and the paraventricular region of the hypothalamus, which is directly related to autonomic regulation [11]. Kc \& Dick [2] highlight that paraventricular nucleus neurons present stress signalizers, which modulate GABA neurotransmitters increasing sympathetic nervous activity. Thus, it is believed that the eucalyptus essential oil has minimized stressor stimulus within the hypothalamic paraventricular region leading to a possible reduction on sympathetic stimulus to the cardiovascular system. In addition, the associated physical exercise could enhance such responses, via baroreceptor reset, reaching the nucleus of the solitary tract $[11,12]$. However, further studies are needed in order to ensure the abovementioned hypotheses regarding the central modulation of the vascular response in induced stress.

The intervention combining aerobic exercise and eucalyptus essential oil increased affective responses regarding pleasure during recovery from sessions and reduced displeasure related to stress. Park et al. [13] demonstrated psychophysiological responses from individuals facing natural and stressor environments, because changes in autonomic responses in different environments would influence the mood of the individuals. These evidences corroborate the Polyvagal Theory which was discussed by Alvarez et al. [24] and presented by Porges [25]. This theory describes the interaction between the autonomic tone and the social engagement. The increased activity of the vagus nerve and the consequent lower sympathetic activation would play an important role in promoting prosocial behavior and emotional regulation [26]. Furthermore, literature has discussed the relationship of the positive affect and exercise intensity during the session. Such interaction can increase practitioner's chances of returning to the exercise program [27], which means a greater adherence and more physical activity in the medium and long term, especially for sedentary people after starting a low intensity exercise program [28]. These findings corroborate that the reduced dose of exercise used in the current study, employing low volume and intensity ( $15 \mathrm{~min} ; \mathrm{RPE}=12)$, contributes to an improved affective feeling in the $\mathrm{AE}+\mathrm{EO}$ post-session stress and recovery (Fig 6). Finally, the interaction of the environment [13] or of the inhaled natural substance [6], besides modulating the mood [29] as observed in the current study regarding affective responses, also reduced the cardiovascular responses during recovery and under stress.

The sample size may be considered as a main limitation of the study. However, a previous sample size calculation suggested the number of subjects investigated in the respective experimental design. Other limitation, this study did not perform measurements of autonomic function, flow-mediated dilation and mood and activation state in recovery and under stress. Such procedures would contribute with additional information to explain the results. It was also not possible to create a placebo substance of eucalyptus essential oil for inhalation in control moments; however, the face mask was kept in those periods and volunteers breathed blank air, as demonstrated by other authors [21].

We concluded that a low dose of aerobic exercise associated with eucalyptus essential oil, as well as the eucalyptus essential oil alone, contributed to reduced responses in the systolic blood pressure reactivity to induced cardiovascular stress. This strategy also enabled a greater sense of pleasure during recovery and less displeasure during stress. Further investigations regarding the interaction of physical exercise with natural essential oils are suggested as an attempt to elucidate both the effects and the possible mechanistic pathways on neurovascular and affective variables.

\section{ACKNOWLEDGEMENTS}

The authors thank FACEPE (Research Support Foundation of the State of Pernambuco) to fund scholarships.

\section{CONFLICTS OF INTEREST}

There is no conflict of interest.

\section{FUNDING}

Authors declared there is no funding or support.

\section{REFERENCES}

1. Chobanian AV, Bakris GL, Black HR, Cushman WC, Green LA, Izzo JL, Jr., et al. The Seventh Report of the Joint National Committee on Prevention, Detection, Evaluation, and Treatment of High Blood Pressure: the JNC 7 report. JAMA. 2003;289(19):2560-72. DOI: 10.1001/jama.289.19.2560 PMID: 12748199

2. Kc P, Dick TE. Modulation of cardiorespiratory function mediated by the paraventricular nucleus. Respir Physiol Neurobiol. 2010;174(12):55-64. DOI: 10.1016/j.resp.2010.08.001 PMID: 20708107

3. Moreira SR, Lima RM, Silva KE, Simoes HG. Combined exercise circuit session acutely attenuates stress-induced blood pressure reactivity in healthy adults. Braz J Phys Ther. 2014;18(1):38-46. PMID: 24675911

4. Atchley RA, Strayer DL, Atchley P. Creativity in the wild: improving creative reasoning through immersion in natural settings. PLoS One. 2012;7(12):e51474. DOI: 10.1371/journal.pone.0051474 PMID: 23251547

5. Gladwell VF, Brown DK, Wood C, Sandercock GR, Barton JL. The great outdoors: how a green exercise environment can benefit all. Extrem Physiol Med. 2013;2(1):3. DOI: 10.1186/2046-7648-2-3 PMID: 23849478

6. Santos MRV, Moreira FV, Fraga BP, Souza DP, Bonjardim LR, Quintans-Junior LJ. Cardiovascular effects of monoterpenes: a review. Braz J Pharm. 2011;21(4):764-71.

7. Lahlou S, Figueiredo AF, Magalhaes PJ, Leal-Cardoso JH. Cardiovascular effects of 1,8-cineole, a terpenoid oxide present in many plant essential oils, in normotensive rats. Can J Physiol Pharmacol. 2002;80(12):1125-31. PMID: 12564637

8. Soares MC, Damiani CE, Moreira CM, Stefanon I, Vassallo DV. Eucalyptol, an essential oil, reduces contractile activity in rat cardiac muscle. Braz J Med Biol Res. 2005;38(3):453-61. DOI:/S0100879X2005000300017 PMID: 15761626

9. Thijssen DH, Maiorana AJ, O’Driscoll G, Cable NT, Hopman MT, Green DJ. Impact of inactivity and exercise on the vasculature in humans. Eur J Appl Physiol. 2010;108(5):845-75. DOI: 10.1007/ s00421-009-1260-x PMID: 19943061

10. Tipton CM. Concerning postexercise hypotension. Exerc Sport Sci Rev. 2011;39(2):109. DOI: 10.1097/JES.0b013e3182164376 PMID: 21430451

11. Chen CY, Bonham AC. Postexercise hypotension: central mechanisms. Exerc Sport Sci Rev. 2010;38(3):122-7. DOI: 10.1097/ JES.0b013e3181e372b5 PMID: 20577060

12. Halliwill JR, Buck TM, Lacewell AN, Romero SA. Postexercise hypotension and sustained postexercise vasodilatation: what happens after we exercise? Exp Physiol. 2013;98(1):7-18. DOI: 10.1113/ expphysiol.2011.058065 PMID: 22872658

13. Park BJ, Tsunetsugu Y, Kasetani T, Hirano H, Kagawa T, Sato M, et al. Physiological effects of Shinrin-yoku (taking in the atmosphere of the forest)--using salivary cortisol and cerebral activity as indicators. J Physiol Anthropol. 2007;26(2):123-8. PMID: 17435354

14. Borg G. Ratings of perceived exertion and heart rates during shortterm cycle exercise and their use in a new cycling strength test. Int 
J Sports Med. 1982;3(3):153-8. DOI: 10.1055/s-2008-1026080 PMID: 7129724

15. Simoes HG, Hiyane WC, Benford RE, Madrid B, Prada FA, Moreira $\mathrm{SR}$, et al. Lactate threshold prediction by blood glucose and rating of perceived exertion in people with type 2 diabetes. Percept Mot Skills. 2010;111(2):365-78. DOI: 10.2466/06.13.15.27.PMS.111.5.365378 PMID: 21162440

16. Santos AS, Alves SM, Figueiredo FJC, Da Rocha Neto OG. Descrição de sistema e de métodos de extração de óleos essenciais e determinação de umidade de biomassa em laboratório. Embrapa East Amazon Tech Announce. 2004

17. Stergiou GS, Tzamouranis D, Protogerou A, Nasothimiou E, Kapralos C. Validation of the Microlife Watch BP Office professional device for office blood pressure measurement according to the International protocol. Blood Press Monit. 2008;13(5):299-303. DOI: 10.1097/MBP.0b013e3283057af6 PMID: 18799957

18. Hines EA, Brown GE. The cold pressor test for measuring the reactibility of the blood pressure: data concerning 571 normal and hypertensive subjects. Am Heart J. 1936;11(1):1-9.

19. Vieira FLH, Lima EG. Laboratorial stress tests and arterial hypertension. Rev Bras Hipertens. 2007;14:98-103.

20. Hardy CJ, Rejeski WJ. Not what, but how one feels: The measurement of affect during exercise. J Sport Exerc Psychol. 1989;11(3):304-17.

21. Robertson RJ, Moyna NM, Sward KL, Millich NB, Goss FL, Thompson PD. Gender comparison of RPE at absolute and relative physiological criteria. Med Sci Sports Exerc. 2000;32(12):2120-9. PMID: $\underline{11128861}$

22. Dayawansa S, Umeno K, Takakura H, Hori E, Tabuchi E, Nagashima $\mathrm{Y}$, et al. Autonomic responses during inhalation of natural fragrance of Cedrol in humans. Auton Neurosci. 2003;108(1-2):79-86. DOI: 10.1016/j.autneu.2003.08.002 PMID: 14614968

23. Li Q, Otsuka T, Kobayashi M, Wakayama Y, Inagaki H, Katsumata $\mathrm{M}$, et al. Acute effects of walking in forest environments on cardiovascular and metabolic parameters. Eur J Appl Physiol. 2011;111(11):2845-53. DOI: 10.1007/s00421-011-1918-z PMID: $\underline{21431424}$

24. Alvares GA, Quintana DS, Kemp AH, Van Zwieten A, Balleine BW, Hickie IB, et al. Reduced heart rate variability in social anxiety disorder: associations with gender and symptom severity. PLoS One. 2013;8(7):e70468. DOI: 10.1371/journal.pone.0070468 PMID: $\underline{23936207}$

25. Porges SW. Orienting in a defensive world: mammalian modifications of our evolutionary heritage. A Polyvagal Theory. Psychophysiology. 1995;32(4):301-18. PMID: 7652107

26. Porges SW. Emotion: an evolutionary by-product of the neural regulation of the autonomic nervous system. Ann N Y Acad Sci. 1997;807:62-77. PMID: 9071344

27. Williams DM, Dunsiger S, Ciccolo JT, Lewis BA, Albrecht AE, Marcus $\mathrm{BH}$. Acute Affective Response to a Moderate-intensity Exercise Stimulus Predicts Physical Activity Participation 6 and 12 Months Later. Psychol Sport Exerc. 2008;9(3):231-45. DOI: 10.1016/j. psychsport.2007.04.002 PMID: 18496608

28. Perri MG, Anton SD, Durning PE, Ketterson TU, Sydeman SJ, Berlant NE, et al. Adherence to exercise prescriptions: effects of prescribing moderate versus higher levels of intensity and frequency. Health Psychol. 2002;21(5):452-8. PMID: 12211512

29. Pretty J, Peacock J, Sellens M, Griffin M. The mental and physical health outcomes of green exercise. Int J Environ Health Res. 2005;15(5):319-37. DOI: 10.1080/09603120500155963 PMID: $\underline{16416750}$ 